\title{
Reabilitação da estética na recuperação da harmonia do sorriso: relato de caso
}

\author{
Rehabilitation of esthetics in recovering smile harmony: case report
}

Paulo Ricardo Barros de Campos*

Dayse Amaral**

Marco Aurélio Chaves da Silva***

Suelem Chasse Barreto ${ }^{* * * *}$

Gisele Damiana da S. Pereira ${ }^{* * *}$

Maíra do Prado ${ }^{* * *}$

\section{Resumo}

Objetivo: este trabalho tem o objetivo de relatar um caso clínico no qual a abordagem restauradora direta com resina composta foi empregada para remodelação estética após tratamento ortodôntico em uma paciente com os incisivos laterais superiores com morfologia conoide. Relato de caso: o paciente, 24 anos, gênero feminino, procurou tratamento odontológico queixando-se da aparência dos incisivos laterais superiores, que se apresentavam fora dos padrões de tamanho e forma devido à existência de uma alteração de estrutura. No exame clínico, constatou-se a presença dos incisivos laterais conoides, dentes 12 e 22. Após o exame radiográfico, que demonstrou ausência de alteração da forma radicular desses elementos, foi planejada a reabilitação funcional e a recuperação estética por meio de restaurações diretas com resina composta. Para reabilitar os elementos estética e funcionalmente, optou-se pela reanatomização, utilizando-se resina composta pela técnica da mão livre, por ser um procedimento mais rápido e de menor custo, por dispensar a etapa de enceramento e moldagem. Todavia, essa técnica apresenta como desvantagem a necessidade de mais habilidade e treinamento do operador. Considerações finais: as resinas compostas permitem uma resolução de maneira rápida e com elevado desempenho estético, apresentando resultado satisfatório, desde que sejam respeitadas suas indicações, compreendidas suas limitações e que a etapa de ajuste oclusal não seja negligenciada.

Palavras-chave: Anatomia. Estética dentária. Resinas compostas. Restauração dentária permanente.

\section{Introdução}

O atual padrão de beleza, sob influência de uma cultura consumista e impulsionado pelos veículos de comunicação, estabelece que o indivíduo é melhor aceito socialmente se apresentar dentes com proporções adequadas, bem alinhados nos arcos e de coloração mais clara, de modo que deformidades no sorriso, adquiridas ou congênitas, podem estabelecer fatores limitantes às relações sociais ${ }^{1}$.

Diante dessas influências e da evolução dos materiais restauradores que possibilitaram uma mimetização mais fidedigna dos elementos dentários, o enfoque da odontologia tradicional, que há mais de um século era apenas de restaurar dentes cariados, foi direcionado para reabilitações estéticas, nas quais restaurações ou recontornos dentários objetivam alterar, associados com a manutenção dos aspectos biológicos e funcionais, a aparência do sorriso, que, muitas vezes, são as causadoras de variações no comportamento do paciente, podendo ser a causa para o desenvolvimento de uma personalidade mais introspectiva ${ }^{2}$.

Dentes conoides são considerados uma microdontia isolada, visto que, em sua maioria, o incisivo lateral superior é mais frequentemente afetado, sendo caracterizado pela apresentação da coroa sob a forma de cone e a raiz comumente com seu comprimento normal. Devido ao seu tamanho reduzido, essa patologia pode representar uma das causas do surgimen-

http://dx.doi.org/10.5335/rfo.v20i2.4436

Especialista, professor, Especialização em Dentística, Clivo Odontologia, Rio de Janeiro, Rio de Janeiro, Brasil.

Especialista, professor, Especialização em Dentística, Clivo Odontologia, Rio de Janeiro,- Rio de Janeiro, Brasil.

Graduada em Odontologia, mestranda em Clínica Odontológica, Faculdade de Odontologia de Piracicaba, Universidade Estadual de Campinas, Piracicaba, São Paulo, Brasil.

Doutora, professora de Clínica Integrada, Faculdade de Odontologia, Universidade Federal do Rio de Janeiro, Rio de Janeiro,-Rio de Janeiro, Brasil. 
to de diastemas anteriores e a correção do problema muitas vezes requer a reanatomização do elemento ${ }^{3}$.

Para tal correção, pode-se lançar mão de resinas compostas, que são materiais que apresentam características estéticas e mecânicas adequadas, associadas ao uso de sistemas adesivos, permitindo, assim, que essas restaurações sejam realizadas com o mínimo ou sem qualquer desgaste à estrutura dental sadia ${ }^{4}$.

Dessa forma, o presente artigo tem como objetivo relatar o caso de um paciente com os incisivos laterais superiores com morfologia conoide, em que uma abordagem restauradora direta com resina composta foi empregada para remodelação estética após tratamento ortodôntico.

\section{Relato de caso}

A paciente leucoderma, de 24 anos, do gênero feminino, compareceu à Clínica de Especialização de Dentística Restauradora, queixando-se da aparência dos incisivos laterais superiores que se apresentavam fora dos padrões de tamanho e forma devido a uma alteração de estrutura. No exame clínico, foi constatada a presença de dois incisivos laterais conoides, dentes 12 e 22 (Figuras 1 e 2). Após o exame radiográfico, que demonstrou ausência de alteração da forma radicular desses elementos, foi planejada a reabilitação por meio de restaurações diretas com resina composta.

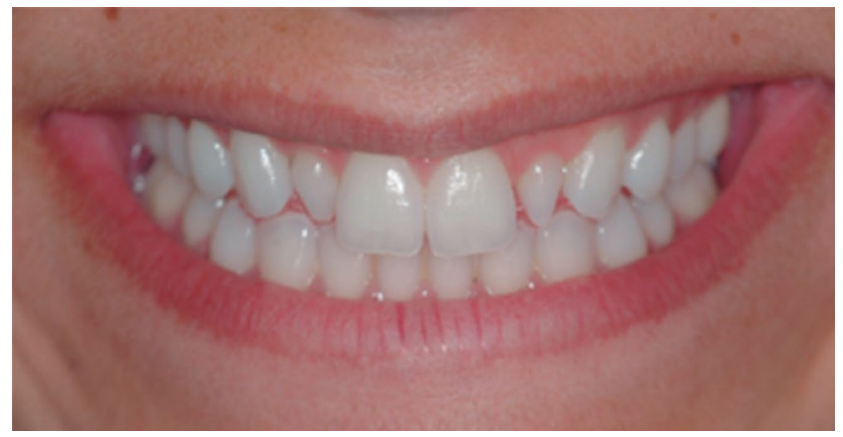

Figura 1 - Foto inicial - vista frontal do sorriso

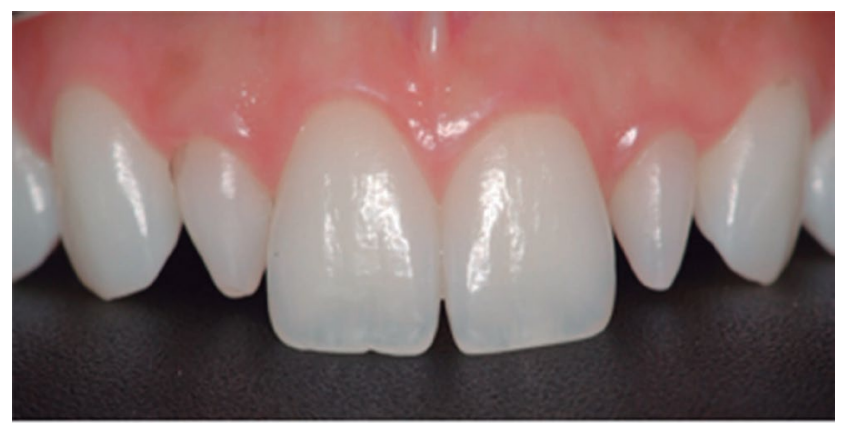

Figura 2 - Avaliação intraoral inicial

Para reabilitar os elementos estética e funcionalmente, optou-se pela reanatomização utilizando resina composta (Opallis-FGM Joinville, Santa Catarina, Brasil), pela técnica da mão livre, por ser um procedimento mais rápido e de menor custo, por dispensar a etapa de enceramento e moldagem, toda- via, tenha a desvantagem de exigir mais habilidade e treinamento do operador.

Para a obtenção dos corretos padrões de tamanho do dente restaurado, foram utilizados os parâmetros estéticos de proporções que incluem: altura do incisivo lateral cerca de $80 \%$ da medida cervico-incisal do incisivo central e largura entre $75 \%$ a $85 \%$ da altura do dente.

Previamente ao procedimento restaurador, foi realizada a profilaxia com pedra pomes e digluconato de clorexidina a $2 \%$, para seleção da cor da resina. Após a escolha de cor, com o auxílio da escala Vita ${ }^{\circledR}$, pequenas porções de resina composta das cores escolhidas foram fotopolimerizadas (fotopolimerizador SmartLite PS Dentsply Petrópolis, Rio de Janeiro, Brasil) por 20 segundos na superfície vestibular dos dentes, sem condicionamento prévio, para aferição da cor com os dentes úmidos. As cores escolhidas para os dentes foram EA2 para esmalte e DA2 para dentina, utilizando ainda resinas de efeito como a T-blue (FGM- Joinville, Santa Catarina, Brasil) para obtenção da caracterização do policromatismo dental.

O mapeamento dental foi realizado definindo a cromaticidade base, o valor e a opalescência. A cromaticidade é a cor da dentina, que deve ser escolhida observando o terço cervical e médio do dente. $\mathrm{O}$ valor é o esmalte, e deve ser feito com o terço médio do dente, enquanto a opalescência tem como base o esmalte presente na incisal dos dentes.

Finalizada a fase de mapeamento de cor, realizou-se o isolamento absoluto com dique de borracha (Madeitex - São José dos Campos, São Paulo, Brasil). Os elementos conoides obtiveram o isolamento travado na cervical com auxílio de uma amarria realizada com fio dental para garantir o correto afastamento gengival, uma vez que esses dentes apresentavam uma inclinação que poderia dificultar a estabilização do lençol de borracha (Figura 3). Esse afastamento possibilitou um perfil proximal mais suave, que resultou em uma adaptação gengival adequada entre os elementos após a conclusão da restauração. A função da amarria é proporcionar um posicionamento mais apical do lençol de borracha.

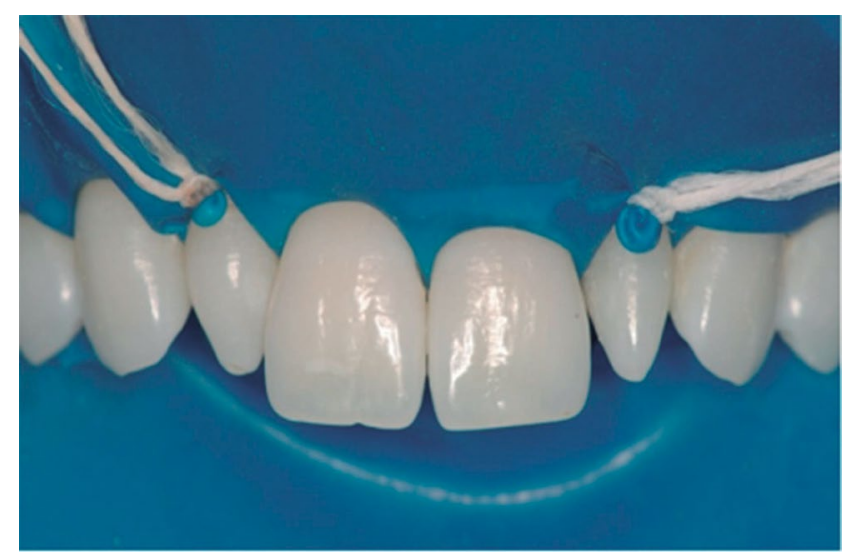

Figura 3 - Isolamento absoluto confeccionado com amarrias 
Uma vez isolados os elementos conoides, a superfície vestibular em esmalte foi condicionada com ácido fosfórico a 37\% (Super Etch- SDI- Pinheiros, São Paulo, Brasil), por 30 segundos, utilizando uma matriz de poliéster (fita matriz poliéster - TDV Joinville, Santa Catarina, Brasil) para o isolamento dos adjacentes (Figura 4). Depois de transcorrido o tempo de condicionamento, realizou-se a remoção do ácido por meio de lavagem abundante com água pelo dobro do tempo do condicionamento. O sistema adesivo Single Bond (3M-ESPE Sumaré, São Paulo, Brasil) foi aplicado conforme as instruções do fabricante e, em seguida, fotopolimerizado.

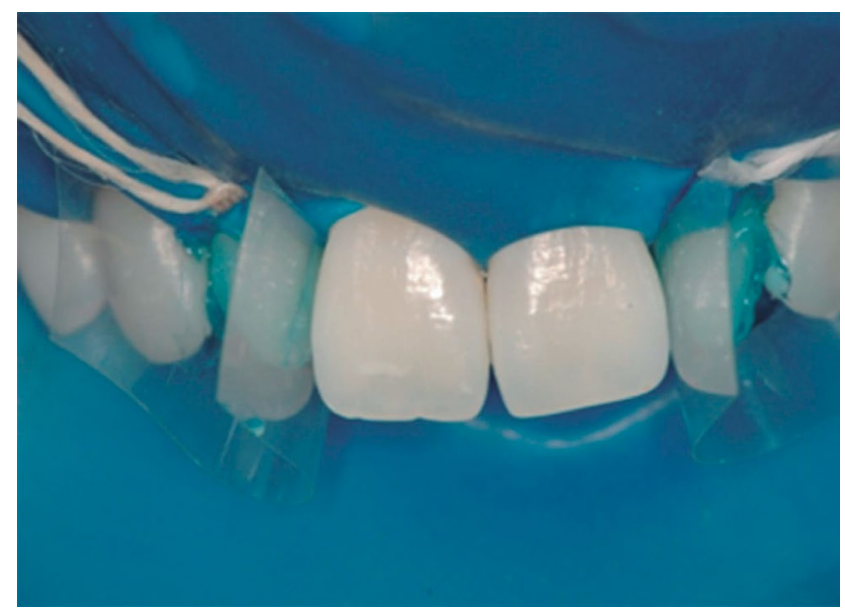

Figura 4 - Matriz de poliéster posicionada

Com auxílio de espátulas Suprafill 1 e 2 (DuflexSswhite - São Cristovão, Rio de Janeiro, Brasil), foi acrescida resina de dentina, o primeiro incremento foi realizado pelas proximais, proporcionando o novo formato do dente, foi delimitado um delgado espaço entre o dente restaurado e o vizinho, que posteriormente foi completado com resina translúcida a fim de proporcionar ao elemento a adequada caracterização das cristas reflexivas (Figura 5).

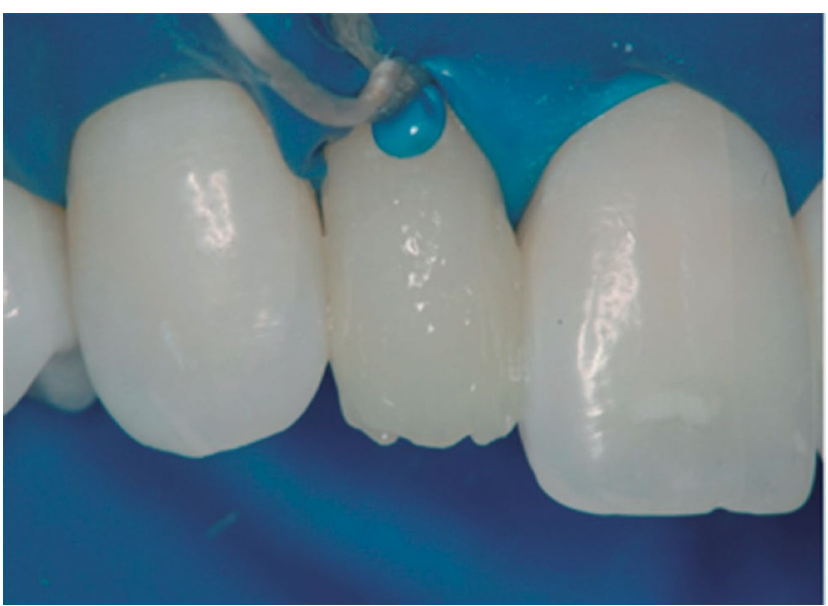

Figura 5 - Início da reanatomização

Em seguida, realizou-se a caracterização do halo incisal com resina translúcida de coloração azulada (T-blue - FGM - Joinville, Santa Catarina, Brasil)
(Figura 6), seguida por um último incremento de resina composta de esmalte, que recobriu os outros incrementos, compondo a área vestibular da restauração (Figura 7). Cada incremento, de aproximadamente $2 \mathrm{~mm}$, foi fotopolimerizado por 40 segundos. A sequência operatória da restauração do incisivo lateral esquerdo foi similar à do direito.

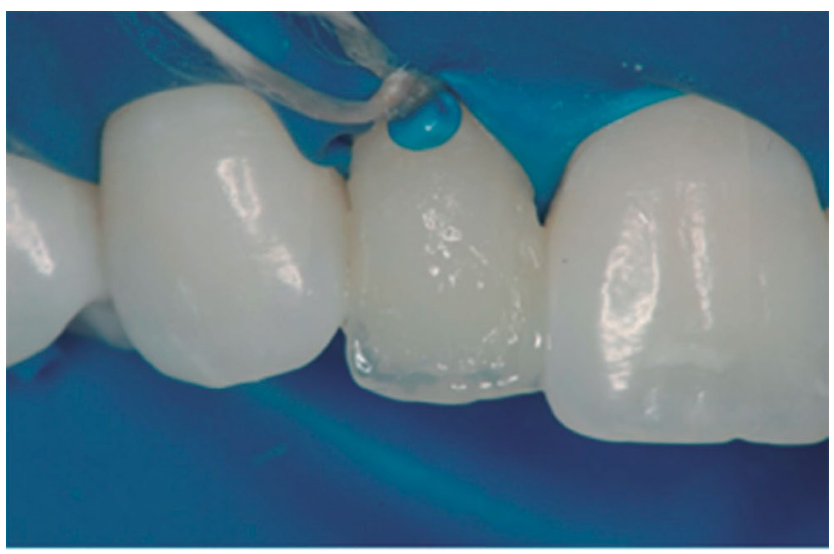

Figura 6 - Halo incisal caracterizado

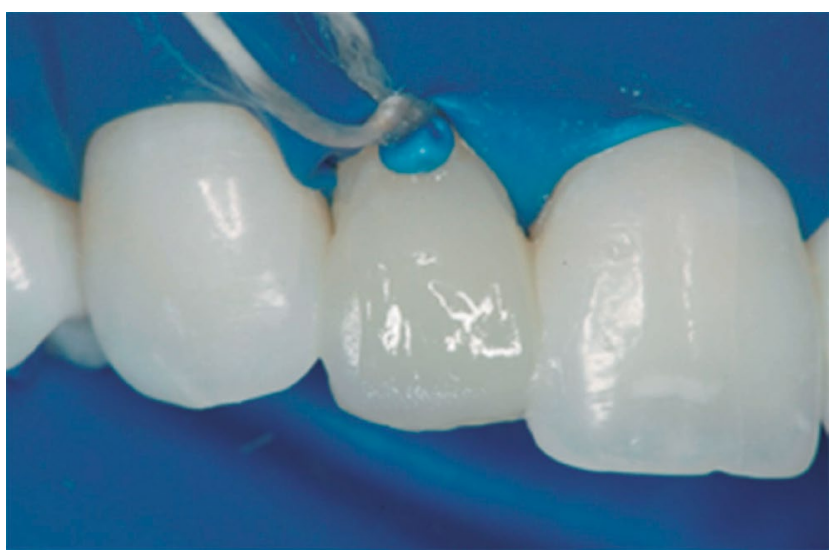

Figura 7 - Incremento final fotopolimerizado

Finalizada a restauração, o acabamento foi iniciado, removendo-se os excessos grosseiros com lâmina de bisturi no 12 (Solidor - Diadema, São Paulo, Brasil). O uso de pontas diamantadas ultrafinas 2200 FF e 3168 FF (KG - Sorensen - Barueri, São Paulo, Brasil) complementou o acabamento e permitiu o ajuste oclusal das áreas demarcadas pelo papel carbono (Accu Film II - Parkell - New York, Estados Unidos). O polimento foi então realizado com borrachas abrasivas de silicone (Jiffy Polisher Ultradent - Curitiba, Paraná, Brasil), seguidas por discos abrasivos de granulação fina (Diamond Pro/ Diamond Flex - FGM - Joinville, Santa Catarina, Brasil), e por disco de feltro (Feltros Diamond FGM - Joinville, Santa Catarina, Brasil) com pasta diamantada (Diamond Ac I e II - FGM - Joinville, Santa Catarina, Brasil), a fim de proporcionar brilho e lisura às restaurações, de tal forma que se conseguisse um aspecto similar à textura do esmalte adjacente (Figura 8), obtendo um aspecto final esteticamente satisfatório. 


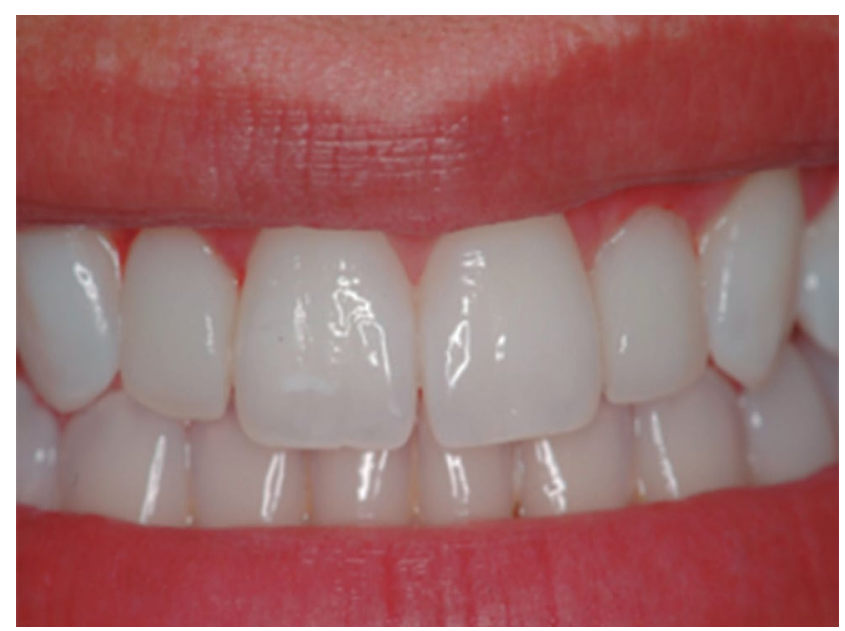

Figura 8 - Vista aproximada da reanatomização e aspecto final do sorriso

Durante a avaliação inicial, notou-se a ausência de guia de desoclusão pelos caninos. Dessa forma, no caso finalizado, pode-se observar o aumento incisal dos caninos, que foi realizado a fim de reabilitar a função oclusal em lateralidade e melhorar a harmonia do sorriso.

\section{Discussão}

A odontologia cosmética apresenta-se como um dos fatores primordiais no planejamento odontológico, influenciando as pessoas, seja por meio da mídia ou da própria sociedade, na busca por obter restaurações imperceptíveis e dentes cada vez mais claros. A harmonia facial depende da disposição, do alinhamento e da posição dos dentes, que, quando se encontram em uma geometria apropriada, traduzem a expressão do que é belo. Contudo, um dos problemas encontrados, e aqui relatado, é o desequilíbrio na harmonia do sorriso causado por alterações promovidas por bordas incisais gastas ou fraturadas e, ainda, ausências dentárias, diastemas, giroversões, apinhamentos e dentes conoides ${ }^{5-7}$.

A discrepância de Bolton ${ }^{8}$ é uma patologia relacionada à anatomia dentária, em que há excesso ou redução do diâmetro mésio-distal dos dentes em relação ao seu homólogo na arcada, ocorrendo em um número considerável de pacientes. Essa discrepância requer modificação da estrutura dentária a fim de obter a similaridade de forma e proporção dos elementos ${ }^{9-11}$. O diastema pode ser definido como sendo o espaço entre os dentes ou a ausência de contato entre dois ou mais dentes consecutivos, podendo apresentar origem patológica ou fisiológica ${ }^{5,12}$. Essa alteração pode estar presente em qualquer local, em ambas as arcadas, sendo uma das causas estéticas mais comuns dos pacientes quando localizado na linha mediana, embora não reduza a eficiência mastigatória $^{13-16}$. O emprego da ortodontia e de próteses fixas foi por muito tempo a alternativa clínica de escolha para solução desses problemas ${ }^{17}$, porém, muitos pacientes não são receptivos ao tratamento ortodôntico por ser um tratamento mais demorado e com custo mais alto ${ }^{18}$. Além disso, muitas vezes, o tratamento ortodôntico isolado não é suficiente para a aproximação ideal dos dentes, sendo necessário, portanto, a correção estética subsequente à ortodontia ${ }^{19}$.

O tratamento ortodôntico é capaz de promover alterações no sorriso do paciente por meio da modificação de inadequadas posições ou inclinações dos elementos dentários, e apresenta como vantagem o fato de ser uma técnica conservadora. Todavia, nem sempre a resolução estética do caso poderá dar-se apenas com a intervenção ortodôntica, já que características individuais dos elementos dentários, como forma, tamanho, e proporção altura/largura ${ }^{8}$, não são alteradas, fazendo-se necessário o inter-relacionamento com a dentística, a periodontia, a implantodontia e a prótese para se obter resultados satisfatórios em estética, função, saúde e estabilidade, como finalizadores do caso ${ }^{5}$.

Nesse contexto, a reformulação dos dentes naturais, utilizando o contorno cosmético, por meio da confecção de restaurações adesivas imperceptíveis e duradouras com compósitos, acompanhadas de biséis incisais e/ou vestibulares, na própria estrutura dental, criando ilusões ópticas, representam uma alternativa simples e eficaz na recuperação de uma aparência mais harmônica, adequando o sorriso aos padrões estéticos desejáveis ${ }^{2}$, como o que foi apresentado neste caso. Assim sendo, alterações como presença de dentes conoides, discrepância de Bolton, contornos dentais inadequados e presença de diastemas por proporções mésio-distais reduzidas dos elementos são situações clínicas que requerem a intervenção restauradora pós-tratamento ortodôntico $0^{6-8}$, que pode ser realizada de forma simples e direta.

O desenvolvimento de novas técnicas e materiais restauradores possibilitaram uma ampliação nas opções de tratamento que podem melhorar a aparência natural dos dentes, de maneira simples, rápida, econômica e conservadora. Sendo assim, pode-se citar como opção para o tratamento restaurador: facetas laminadas em cerâmica; uso de resina composta e, muitas vezes, associação gengivectomia/gengivoplastia, nos casos em que há a necessidade de alongar a coroa no sentido cérvico-incisal dos elementos dentários $^{10-14}$. A correta obtenção dos aspectos de brilho e sombra nos dentes é muito importante, uma vez que em uma restauração, seguindo uma ordem de importância das dimensões da cor, têm-se: valor, translucidez, croma e matiz. De forma que o valor é três vezes mais importante que o matiz, e duas vezes mais que o croma. Sendo assim, o valor é a mais importante dimensão da cor dos dentes. Isso decorre do fato de essa dimensão ser percebida pelo olho humano com maior acuidade que as demais, e com isso cores distintas de mesmo valor podem parecer idênticas. Ainda, não se pode esquecer a opacidade e translucidez, consideradas a quarta dimensão da cor.

É fato que a composição atual dos compósitos apresenta características mecânicas e estéticas de excelência, o que proporciona durabilidade satisfatória e favorece ainda mais seu emprego nas restaurações diretas. Porém, é de suma importância a ava- 
liação dos movimentos excursivos, pois a existência de uma oclusão balanceada com funcionamento das guias de desoclusão é essencial para a manutenção de uma adequada longevidade da restauração.

\section{Considerações finais}

Diante do exposto pôde-se concluir que:

a) a reanatomização dentária para a finalização de casos ortodônticos pode ser necessária em casos que requerem mudança em proporções dos elementos;

b) as resinas compostas possibilitam a confecção da restauração de forma direta, por meio da utilização de um material com adequado custo-benefício e excelentes resultados estéticos, e de longevidade satisfatória quando bem empregadas;

c) estas restaurações possibilitam um resultado estético satisfatório, com uso de uma técnica que não requer desgastes teciduais, já que sua retenção é obtida pela utilização dos sistemas adesivos.

\section{Abstract}

Objective: This study aimed to report a clinical case where a direct restorative composite resin approach was applied for esthetic remodeling, after orthodontic treatment of a patient with conoid-shaped upper lateral incisors. Case report: Patient, 24 years old, female gender, sought dental treatment complaining about the appearance of upper lateral incisors that did not match parameters of size and shape, because of a change in structure. On clinical examination, it was observed the presence of the lateral conoid incisors - teeth 12 and 22. After radiographic examination, which showed no change in root shape for these elements, their functional rehabilitation and esthetic recovery was planned through direct restorations with composite resin. In order to esthetically and functionally rehabilitate the elements, re-anatomization with composite resin by the freehand technique was chosen, because it is a faster and less expensive procedure, and eliminates the finishing and molding step. However, this technique presents the disadvantages of requiring higher skills and trained operators. Final considerations: Composite resins allow quick resolution with high esthetic performance, so the result is satisfactory upon respecting recommendations, understanding limitations, and not neglecting the occlusal adjustment step.

Keywords: Anatomy. Dental esthetics. Composite resins. Permanent dental restoration.

\section{Referências}

1. Cardoso RM, Maia GHS, Melo CA, Guimarães RP, Menezes Filho PF. Aplicação clínica das resinas de nanotecnologia. Odontol Clin Cient 2008; 7(4):357-62.

2. Pfeifer JMG, Nascimento F, Soares CJ, Oliveira LCAD, Abdalla MC. Conceitos de estética envolvidos no fechamento de diastemas e reanatomização de dentes anteriores com resina composta. JBD Rev Ibero-am Odontol Estetica \& Dentistica 2004; 3(10):122-31.
3. Neville B, Bouquot JE, Damm DD, Allen CM. Patologia oral e maxilofacial. 3. ed. Rio de Janeiro: Elsevier, 2009. 972 p.

4. Shimomura M, Egoshi M, Cruz F, Grattão I. Fechamento de diastema: técnica alternativa utilizando resinas compostas. Rev Associação Odontológica do Norte do Paraná, 2002; 12:6-10.

5. Kreia TB. O dilema dos diastemas interincisivos superiores em ortodontia. Nova visão em ortodontia e ortopedia funcional dos maxilares. São Paulo: Livraria Ed. Santos; 2002 p. 907-11.

6. Freeman JE, Maskeroni AJ, Lorton L. Frequency of Bolton tooth-size discrepancies among orthodontic patients. Am J Orthod Orthop 1996; 110(1):24-7.

7. Miller WB, Mclendon WJ, Hines FB. Two treatment approaches for missing or peg-shaped maxillary lateral incisors: a case study on identical twins. Am J Orthod Dentofac Orthop 1987; 92(3):249-56.

8. Ramos AL, Suguino R, Terada HH, Furquim LZ, Da Silva Filho OZ. Considerações sobre análise da discrepância dentária de Bolton e a finalização ortodôntica. Rev Dental Press Ortodon Ortop Facial 1996; 1(2):86-106.

9. Bolton W. Disharmony in tooth size and its relation to the analysis and treatment of maloclusion. AJO 1958; 28(3):113-30.

10. Kalix AP, Maia RR, Vargas E, Amaral D, Capelli Júnior J. Reanatomização de dentes ântero-superiores. Rev Bras Odontol 2003; 60(1):18-20.

11. Mandarino F, Machado CT, Andrade MF, Flores VO. Possibilidades de resoluções clínicas oferecidas pelas técnicas de odontologia cosmética associada a materiais estéticos. J Bras Odontol Clin 1998; 2(10):12-5.

12. Mondelli RFL, Lopes LG. Estabelecimento da harmonia estética do sorriso associado às técnicas de clareamento e reanatomização dental para finalização de tratamento ortodôntico - relato de caso. J Bras Clin Estet Odontol 2000; 4(23):84-9.

13. Morley J, Eubank J. Macroesthetic elements of smile design. J Am Dent Assoc 2001; 132:39-45.

14. Omais S, Yassumoto M. Reanatomização e recontorno cosmético de dentes anteriores - relato de caso clínico. J Bras Clin Odontol Int 2001; 5(30):499-502.

15. Bishara SE. Management of diastemas in orthodontics. Am J Orthod 1972; 61(1):55-63.

16. Cangialossi TJ. Management of maxillary central incisor impacted by a supernumerary tooth. J Am Dent Assoc 1982; 105(5):812-4.

17. Almeida RR, Garib DG, Almeida-Pedrin RR, Almeida MR, Pinzan A, Junqueira MHZ. Diastemas interincisivos centrais superiores: quando e como intervir? Rev Dental Press Ortodon Ortop Facial 2004; 9(3):137-56.

18. Fortkamp S, Araújo Junior EM, Baratieri LN. Indução papilar em dentes anteriores com diastemas por meio de restaurações adesivas: relato de um caso clínico. Clínica - Int J Braz Dent 2005; 1:127-35.

19. Chimeli TBC, Pedreira APRV, Souza TCP, Maciel M, Paula LM, Garcia FCP. Tratamento restaurador de diastemas anteriores com restaurações diretas em resina composta: relato de caso. Rev Dentistica On-line 2011; 12(20):54-7.

\section{Endereço para correspondência:}

Marina Sousa Azevedo

Rua Gonçalves Chaves 457, $7^{\circ}$ andar, sala 704

96015-560 Pelotas, RS

Telefone: 32224305 ramal 127

E-mail: marinasazevedo@hotmail.com

Recebido: 22/10/14. Aceito: 22/06/15. 